\title{
A Comparative Assessment of the EIA/PCR HIV Test Results among Weakly Reactive Samples on Any of Determine HIV1/2, Statpak and Unigold Rapid Test Kits
}

Kigozi D Ssebaggala1*, Lutalo Tom ${ }^{1}$, Maria J Wawer ${ }^{1,2}$, Sserwadda David ${ }^{1,3}$, Steven J Reynolds ${ }^{1,4}$, Nalugoda Fred ${ }^{1}$, Kigozi Godfrey ${ }^{1}$, Ronald H Gray ${ }^{1,2}$, Kalibbala Sarah ${ }^{1}$ and Muwanika Richard ${ }^{1}$

${ }^{1}$ Rakai Health Sciences Program, Uganda

${ }^{2}$ Johns Hopkins University, USA

${ }^{3}$ Makerere University School of Public Health, Uganda

${ }^{4}$ National Institute of Allergy and Infectious Diseases, USA

\begin{abstract}
Objective: The Government of Uganda recommends the serial HIV rapid testing algorithm where weakly reactive results on any of the three: Determine, Statpak and Unigold are interpreted as positive. We assessed the proportion of weak positive (WP) rapid results against confirmed results.

Methods: We generated cross tabulations using Stata 3.0 to compare the WP rapid test results on each kit in the algorithm against final results on EIA/PCR to assess the proportion of samples confirmed to be HIV negative on ELISA and/or qualitative DNA PCR. We also generated Positive Predictive Values (PPV) to find the proportion of results that were true positives.
\end{abstract}

Results: A total of 231 weak positive (WP) results were selected. 71 were WP on Determine HIV1/2, 149 on Statpak and 11 on Unigold. We found that $35.2 \%$ of the WP results on Determine were confirmed Negative by EIA/PCR test with a Positive Predictive Value (PPV) of $64.8 \%, 80.5 \%$ of WP on Statpak were confirmed negative with a PPV of $19.5 \%$ while $63.6 \%$ of the WP on Unigold were confirmed negative with a PPV of $36.4 \%$. When using the National serial rapid HIV testing algorithm, WP on both Determine and Statpak are scored as positive. However, we found that out of the 11 WP on both Determine and Statpak for screening and confirmatory tests respectively, $45.5 \%$ returned a final negative result with a PPV of $54.5 \%$.

Conclusion: Since point of care results are interpreted following manufacturer's instructions, a substantial proportion that would have been scored as positive end up with Negative results. WP screening results whether using parallel or serial testing algorithm, need to be confirmed by EIA or PCR before results are finalized. This calls for a reevaluation of the national algorithm that considers WP scores instead of what is instated in the kit insert.

Keywords: Weakly reactive HIV tests; Rapid HIV test; Qualitative PCR test; ELIS/Western blot; Serial testing algorithm; Parallel testing algorithm; Positive predictive value

\section{Introduction}

HIV testing can be conducted for diagnosis, surveillance or blood screening [1] and the selection of HIV testing approaches vary between country's policies.

HIV counseling and testing (HCT) based upon point of care rapid testing is crucial for improving awareness and initiating treatment. Other methods such as ELISA or WB and qualitative PCR are more expensive and complex, requiring laboratory facilities [2,3]. In SubSaharan Africa, it is estimated that less than $40 \%$ of persons know their HIV status [4].

There are two common algorithms for HIV rapid testing namely serial and parallel, however serial is recommended for public health use while parallel is reserved for research settings $[5,6]$.

\section{Serial testing algorithms}

In serial testing, two different rapid tests are applied one after another. Each blood sample is subjected to one rapid test (screening test), and if it is negative, it is reported as HIV negative. But if the test result is positive, it is subjected to a second rapid test (confirmatory test). If the second rapid test is positive the blood sample is reported as positive. Samples that show positive results on the screening test and negative on the confirmatory test should be subjected to a nationally approved third test (tie-breaker). The outcome on the tie breaker is declared as final test result.

\section{Parallel testing algorithms}

Parallel testing means that two different rapid HIV tests are applied concurrently (in parallel) to the same blood sample. Samples which show HIV- positive results on both tests are reported as positive while samples which show HIV-negative results on both tests are reported as HIV negative. Samples which show positive results on one test and negative on the other are subjected to a third test (tie breaker test). The outcome on the tie breaker is recorded as the final test result.

*Corresponding author: Darix Ssebaggala Kigozi, Rakai Health Sciences Program, Uganda; Tel: 256 754106565; E-mail: kdssebaggala@rhsp.org; kdssebaggala@gmail.com

Received December 07, 2016; Accepted February 23, 2017; Published March 02, 2017

Citation: Ssebaggala KD, Tom L, Wawer MJ, David S, Reynolds SJ, et al. (2017) A Comparative Assessment of the EIA/PCR HIV Test Results among Weakly Reactive Samples on Any of Determine HIV1/2, Statpak and Unigold Rapid Test Kits. J AIDS Clin Res 8: 670. doi: 10.4172/2155-6113.1000670

Copyright: (C) 2017 Ssebaggala KD, et al. This is an open-access article distributed under the terms of the Creative Commons Attribution License, which permits unrestricted use, distribution, and reproduction in any medium, provided the original author and source are credited. 
The Ugandan Ministry of Health (MOH) recommends a testing algorithm in which Determine HIV1/2 is used as the first screening test, If this initial test is positive, Statpak is used as the confirmatory test and Unigold is used as a tie breaker in case of discordant results. Samples scoring as Weak Positive (WP) on both Determine and Statpak are considered as positive.

We assessed this algorithm by comparing WP results on all three rapid tests with results of ELISAs (EIA) or/and qualitative DNA PCR to find out the proportion of the samples that test positive. Previous studies revealed that challenges to rapid test include test sensitivity and the subjective interpretation of weakly reactive results at the point of care [7]. The Ugandan $\mathrm{MOH}$ defines a sample to be reactive if two lines of any intensity of bands appear in both the control and the patient area. This is clearly stated in the Standard Operating Procedure (SOP) for Health laboratory services by Central Public Health Labs first edition Nov, 2008 (Unpublished).

\section{Location}

The study was conducted in Rakai district which is located in southern Uganda.

\section{Sample size}

A purposive sample from 231 sample test results was selected from the Rakai Community Cohort Study (RCCS) because they had a WP result on Determine, Statpark or Unigold between July, 2013 and April 2015. This included 71 WP results on Determine, 149 WP results on Statpack and $11 \mathrm{WP}$ results on Unigold.

\section{Inclusion criteria}

- A selected result must have a weak positive result on one or more rapid tests.

- The sample must have a final result confirmed by ELISA or qualitative DNA PCR.

\section{Testing procedure}

At the point of care, a parallel testing algorithm on rapid HIV using Determine and Statpak is followed where concordant positive or negative results end the testing, while discordant results are further tested on Unigold as a tie-breaker.

All results scored as weakly reactive (With bands of low intensity) are subjected to two ELISA. The final results were confirmed if ELISA results were concordant. In case of discordance between the two ELISAs, a qualitative DNA PCR test was performed as the gold standard.

\section{Results and Discussion}

We generated cross tabulations of the sero-rapid test results against the confirmed EIA/PCR test results to assess the proportion of weak positives that were positive or negative on EIA/PCR. The analysis was done to evaluate independently the proportion of WP results on Determine, Statpak or Unigold that returned Negative results. Additionally, we evaluated the WP results on both Determine and Statpak which were confirmed by EIA/PCR. This is because Samples scoring as WP on both Determine and Statpak would eventually score as positive. The results are as seen in Tables 1 and 2 below.

\section{Weakly reactive results on determine}

Out of the 71 weakly reactive rapid HIV test results on Determine, $35.2 \%$ were confirmed to be HIV Negative (Table 1). The positive Predictive value (PPV) was $64.8 \%$.

\begin{tabular}{|c|c|c|c|c|c|c|c|c|}
\hline Confirmed & & Determine & & Statpak & & Unigold & & Totals \\
\hline HIV results & $\mathbf{N}$ & $\%$ & $\mathbf{n}$ & $\%$ & $\mathbf{n}$ & $\%$ & $\mathbf{N}$ & $\%$ \\
\hline Positive & 46 & 64.8 & 29 & 19.5 & 4 & 36.4 & 79 & 34.2 \\
\hline Negative & 25 & 35.2 & 120 & 80.5 & 7 & 63.6 & 152 & 65.8 \\
\hline Total & 71 & 100.0 & 149 & 100.0 & 11 & 100.0 & 231 & 100.0 \\
\hline
\end{tabular}

Table 1: Weakly reactive samples on the three rapid testing kits assessed on ELISA/ Qualitative PCR.

\begin{tabular}{|c|c|c|}
\hline Confirmed HIV Results & \multicolumn{2}{|c|}{ Determine and Statpak } \\
\cline { 2 - 3 } & N & $\%$ \\
\hline Positive & 6 & 54.5 \\
\hline Negative & 5 & 45.5 \\
\hline Total & 11 & 100.0 \\
\hline
\end{tabular}

Table 2: Weakly reactive samples on both Determine and Statpak testing kits assessed on ELISA/qualitative PCR.

\section{Weakly reactive results on statpak}

From this study, out of the 149 sample with Weak Positive HIV results on Statpak, $80.5 \%$ were confirmed to be Negative as shown in Table 1 and the PPV was $19.5 \%$.

\section{Weakly reactive results on Unigold}

As seen in Table 1 above, out of $11 \mathrm{WP}$ samples on Unigold, 63.6\% were confirmed to be HIV negative and PPV was $36.4 \%$.

\section{Weakly reactive results on both determine and Statpak}

The final results of ELISA/PCR for the weakly reactive samples on both Determine and Statpak revealed that $45.5 \%$ were actually negative and the PPV was $54.5 \%$ as shown in Table 2 below.

\section{Conclusion}

This study shows a high potential for false positive results from point of care since rapid tests are interpreted as per manufacturer's insert.

Precious studies have shown that Determine has a low specificity (85.2\%, positive predictive value $67.3 \%$ ) due to false-positive results with weak-positive bands [8]. This result is in agreement with the findings from this study in which $35.2 \%$ of the WP results were confirmed to be HIV negative and PPV was $64.8 \%$.

It is recommended that the tie-breaker algorithm should be abandoned in favor of WHO recommended serial or parallel algorithms and to interpreted the weakly reactive samples as indeterminate results $[6,9]$. For all the three test kits with WP assessed in this study, we concur with this recommendation since WP bands were disproportionately negative especially with Statpak.

The $\mathrm{MOH}$ algorithm can therefore be improved upon by considering the intensity of the bands/bars while interpreting HIV positive results on all HIV rapid test kits other than presence of any band/intensity as indicated by the $\mathrm{MOH}$.

In another study conducted in the Rakai district of Uganda, when weakly reactive samples were considered as positive, the sensitivity was suboptimal (97.4\%-95\% CI: 96.1-98.4) [5].

Whenever there is a WP result on rapid test screening using either the parallel or serial testing algorithm, a PCR/EIA test should be run to confirm the HIV results. The current Ministry of Health policy which classifies WP results on rapid test kits as positive should be considered as indeterminate. 
Citation: Ssebaggala KD, Tom L, Wawer MJ, David S, Reynolds SJ, et al. (2017) A Comparative Assessment of the EIA/PCR HIV Test Results among Weakly Reactive Samples on Any of Determine HIV1/2, Statpak and Unigold Rapid Test Kits. J AIDS Clin Res 8: 670. doi: 10.4172/21556113.1000670

Page 3 of 3

This research indicates that up to $35.2 \%$ of WP results on Determine and $80.5 \%$ of WP on Statpak are HIV negative. Therefore, Statpak is a weaker test kit to confirm positive (or WP) results on Determine. Where results scored as WP on both Determine and Statpak, the PPV was $54.5 \%$.

There is need to evaluate this national algorithm so that all samples that score as WP on rapid HIV test kits are confirmed by ELISA/PCR before results are issued to clients.

\section{Limitations}

The study was based on small numbers. However, the results suggest that rapid test algorithms require additional validation and potentially a redesign, as well as confirmation by EIA or PCR, in order to avoid issuing out false positive HIV results.

\section{Acknowledgement}

The successful accomplishment of this work was supported by: The National Institute of Health (NIH), The National Institute of Allergy and Infectious Diseases the Gates Foundation under Grant number 22006.03 and the Division of Intramura Research.

\section{Ethics Statement}

The study was approved by the Western IRB, the Uganda Virus Institute Research and Ethics Commitee and the Uganda National Council for Science and Technology.

\section{References}

1. UNAIDS/WHO (2001) Guidelines for using HIV testing technologies in Surveillance: Selection, evaluation and implementation. UNAIDS/WHO, Geneva

2. Tung CS, Sangi-Haghpeykar H, Levison J (2010) Rapid versus standard testing for prenatal HIV screening in a predominantly Hispanic population. $J$ Perinatol 30: 30-32.

3. Delaney KP, Branson BM, Uniyal A, Phillips S, Candal D, et al. (2011) Evaluation of the performance characteristics of 6 rapid HIV antibody tests. Clin Infect Dis 52: 257-263.

4. Anand A, Shiraishi RW, Bunnell RE, Jacobs K, Solehdin N, et al. (2009) Knowledge of HIV status, sexual risk behaviors and contraceptive need among people living with HIV in Kenya and Malawi. AIDS 23: 1565-1573.

5. Galiwango RM, Musoke R, Lubyayi L, Ssekubugu R, Kalibbala S, et al. (2013) Evaluation of current rapid HIV test algorithms in Rakai, Uganda. J Virol Methods 192: 25-27.

6. UNAIDS/WHO (2010) Guidelines for using HIV testing technologies in Surveillance: Selection, evaluation and implementation.

7. Gray RH, Makumbi F, Serwadda D, Lutalo T, Nalugoda F, et al. (2007) Limitations of rapid HIV-1 tests during screening for trials in Uganda: Diagnostic test accuracy study. BMJ 335: 188.

8. Kagulire SC, Opendi P, Stamper PD, Nakavuma JL, Mills LA, et al. (2011) Field evaluation of five rapid diagnostic tests for screening of HIV-1 infections in rural Rakai, Uganda. Int J STD AIDS 22: 308-309.

9. Leslie S, Ruby S, Kliescikova J, Neil P, Cono A, et al. (2015) Evaluation of HIV testing algorithms in Ethiopia: The role of the tie-breaker algorithm and weakly reacting test lines in contributing to a high rate of false positive HIV diagnoses. BMC Infect Dis 15: 39 